Revista Brasil. Bot., V.31, n.4, p.575-586, out.-dez. 2008

\title{
Composição florística de remanescente de cerrado sensu stricto em Botucatu, SP ${ }^{1}$
}

\author{
KATIA LOSANO ISHARA², GUILHERME FERNANDO GOMES DÉSTRO ${ }^{3}$, \\ RITA C. S. MAIMONI-RODELLA ${ }^{2,4}$ e YURIKO A. N. P. YANAGIZAWA ${ }^{2}$
}

(recebido: 23 de março de 2006; aceito: 31 de julho de 2008)

\begin{abstract}
Floristic composition of a "cerrado" sensu stricto remnant in Botucatu, SP). A floristic survey of a "cerrado" sensu stricto area was carried out in Botucatu, west-central region of São Paulo State, southeastern Brazil. One-hundredseventy-nine angiosperms taxa (177 species) were recorded distributed in 122 genera and 49 families. Five species of pteridophytes were also collected, belonging to four genera and two families. The woody component was predominant, representing more than $60 \%$ of the registered species. The arboreal to non-arboreal species ratio was 1:3. The families with the highest species richness were: Asteraceae (27 species), Fabaceae (22), Myrtaceae (11). The comparison among six cerrado areas revealed great floristic heterogeneity since the similarity indexes produced low values, even among neighboring areas.
\end{abstract}

Key words - "cerrado" floristic, profile diagram, similarity

RESUMO - (Composição florística de remanescente de cerrado sensu stricto em Botucatu, SP). Realizou-se o levantamento florístico de uma área remanescente de cerrado sensu stricto no Município de Botucatu, região centro-oeste do Estado de São Paulo, Sudeste do Brasil. Foram registrados 179 táxons de angiospermas (177 espécies) distribuídas em 122 gêneros e 49 famílias e cinco espécies de pteridófitas pertencentes a quatro gêneros e duas famílias. O componente lenhoso foi predominante representando mais de $60 \%$ das espécies registradas. A proporção das espécies arbóreas para as não arbóreas foi de 1:3. As famílias com maior riqueza foram: Asteraceae (27 espécies), Fabaceae (22), Myrtaceae (11). A comparação entre seis áreas de cerrado revelou grande heterogeneidade florística uma vez que os índices de similaridade produziram valores baixos, mesmo entre áreas próximas.

Palavras-chave - diagrama de perfil, florística do cerrado, similaridade

\section{Introdução}

O Cerrado constitui o segundo maior bioma brasileiro, apresentando uma área nuclear localizada no Brasil Central e também algumas penínsulas e áreas disjuntas periféricas, como é o caso do Estado de São Paulo (Ribeiro \& Walter 1998, Durigan et al. 2004a). Neste Estado, a vegetação de cerrado apresenta-se fragmentada, situando-se principalmente na Depressão Periférica e no Planalto Central, além de algumas áreas no Vale do Paraíba (Durigan et al. 2004a). Mesmo possuindo apenas fragmentos de cerrado, estima-se que ocorram em São Paulo cerca de 34\% das espécies arbóreas assinaladas para essa formação (Leitão Filho 1992).

1. Parte da dissertação de mestrado do primeiro autor, Programa de Pós-Graduação em Ciências Biológicas - Botânica da Universidade Estadual Paulista, Instituto de Biociências de Botucatu.

2. Universidade Estadual Paulista, Instituto de Biociências de Botucatu, Departamento de Botânica, 18618-000 Botucatu, SP, Brasil.

3. Universidade Estadual Paulista, Programa de Pós-Graduação em Agronomia - Energia na Agricultura da Faculdade de Ciências Agronômicas de Botucatu.

4._Autor para correspondência: rita@ibb.unesp.br
Até metade do século XX, o Cerrado ocupava 14\% do território paulista; atualmente, os remanescentes dessa vegetação cobrem menos de $1 \%$, devido, principalmente, ao intenso e rápido desmatamento e à falta de políticas de preservação (Durigan et al. 2004a). O Município de Botucatu localiza-se na porção centro-oeste do Estado de São Paulo, inserindo-se, do ponto de vista fisiográfico, na região das Cuestas Basálticas, caracterizada por escarpas e relevos de transição que demarcam a passagem da Depressão Periférica para o Planalto Ocidental Paulista (Spirandelli-Cruz 2004). Os remanescentes de vegetação natural ocorrem no município de forma fragmentada, encontrando-se áreas de floresta estacional semidecidual, florestas ripárias, cerradão e cerrado (Jorge \& Moreira 2000). Estima-se que o município apresente atualmente menos de $20 \%$ de sua superfície ocupada por estas formas de vegetação nativa (Campos et al. 2004).

Diversos levantamentos florísticos e fitossociológicos foram realizados nos cerrados paulistas incluindo o componente arbustivo-arbóreo e/ou o componente herbáceo-subarbustivo da vegetação (Cavassan 2002). Apesar disso, há poucos estudos disponíveis para a o Município de Botucatu e cidades limítrofes, podendo-se 
citar Silberbauer-Gottsberger \& Eiten (1987), que realizaram estudo fitossociológico em um hectare de cerrado e analisaram a distribuição espacial de algumas espécies nessa área, e o de Bicudo (1987), que mapeou as áreas de cerrado, remanescentes no Município de Botucatu, realizando também o estudo florístico em duas áreas selecionadas, sendo uma de cerradão e outra de campo cerrado. Posteriormente, outra área de cerradão nessa região foi estudada por Bicudo (1995), que realizou o levantamento florístico, fitossociológico e análise da ciclagem de nutrientes. Bicudo et al. (1996) compararam a flora arbustivo-arbórea de uma área de cerradão em Botucatu com outras áreas do Estado, encontrando maior similaridade da área estudada com uma de Mogi-Mirim; concluíram que este resultado pode ser decorrente de semelhanças dos fatores edáficos, fisiográficos e graus de proteção ambiental dos distintos locais analisados.

O objetivo do presente trabalho foi contribuir para o conhecimento da vegetação, presente no Município de Botucatu, através da análise da composição florística de um remanescente de cerrado sensu stricto e também comparar essa área com outras já conhecidas no Estado de São Paulo.

\section{Material e métodos}

O estudo foi realizado em um fragmento de cerrado sensu stricto pertencente à empresa Centroflora - Anidro

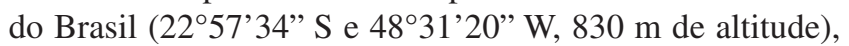
Município de Botucatu, região centro-oeste do Estado de São Paulo. A área de estudo compreende cerca de 5 ha, sendo constituída por seis sub-áreas não contínuas localizadas dentro do espaço ocupado pela empresa. Essas áreas estão limitadas pela rodovia Eduardo Zuccari, pelas instalações da empresa (prédios, galpões e hortas) e por fragmentos de floresta estacional semidecidual. Tanto a vegetação de cerrado quanto a floresta estacional semidecidual estão circundadas por plantações de eucalipto e pastagens dos terrenos vizinhos. O solo da área é do tipo Latossolo Vermelho-Amarelo (Embrapa 1999). O clima da região foi classificado como Cfa de Koeppen (Campos et al. 2004).

O levantamento florístico foi realizado no período de março de 2004 a abril de 2005, coletando-se, semanalmente, plantas em fase reprodutiva, por meio de caminhadas aleatórias por toda a área de cerrado. O material coletado foi prensado no local e, posteriormente, processado para herborização e depositado no Herbário "Irina Delanova de Gemtchújinicov" (BOTU), do Instituto de Biociências da Unesp, campus de Botucatu.

Para a identificação, foram utilizadas bibliografia especializada, comparação com exsicatas do Herbário BOTU e envio para especialistas do Herbário UEC (Instituto de Biologia, Unicamp). Adotou-se o sistema de classificação do
APG II conforme Souza \& Lorenzi (2005) para fanerógamas; para pteridófitas adotou-se o sistema de Tryon \& Tryon (1982).

A fisionomia da vegetação foi caracterizada, elaborandose um diagrama de perfil a partir de uma linha de $30 \mathrm{~m}$ de comprimento por $5 \mathrm{~m}$ de largura, localizada no centro da área amostrada. Os indivíduos presentes nessa faixa, pertencentes ao componente arbustivo-arbóreo, foram mensurados quanto à altura e diâmetros do caule e da copa, mapeados ao longo do perfil e identificados, conforme Durigan (2003).

Para a avaliação da similaridade florística, entre a área do presente estudo e outras áreas de cerrado sensu lato no Estado de São Paulo, foi elaborada uma matriz de presença/ ausência e calculados os Índices de Jaccard e de Sørensen (Mueller-Dombois \& Ellenberg 1974) para possibilitar comparação com a literatura. Foram comparadas apenas as áreas de cerrado nas quais os levantamentos florísticos incluíram todos os hábitos. Foi elaborada análise de agrupamento, utilizando-se o Índice de Jaccard como medida de similaridade e o algoritmo UPGMA para a elaboração do dendrograma (Sneath \& Sokal 1973). É importante ressaltar que, para a comparação entre as áreas, desconsideraram-se as espécies com identificação incompleta (apenas gênero, aff. ou $c f$.), excluindo-se também aquelas cuja ocorrência foi assinalada para apenas uma área, segundo metodologia adotada por Ratter et al. (2003).

\section{Resultados}

$\mathrm{Na}$ área de estudo foram encontrados 184 táxons, sendo 177 espécies de angiospermas distribuídas em 122 gêneros e 49 famílias, e cinco espécies de pteridófitas pertencentes a quatro gêneros e duas famílias (tabela 1).

As famílias mais ricas foram Asteraceae (27 espécies), Fabaceae (22) e Myrtaceae (11) representando $34 \%$ das espécies registradas. Compondo Fabaceae, as subfamílias Faboideae, Caesalpinioideae e Mimosoideae apresentaram, respectivamente, onze, sete e quatro espécies. Entre os gêneros mais ricos estão: Vernonia (oito espécies), Solanum (sete), Eupatorium (seis), Erythroxylum e Myrcia (quatro espécies cada). Famílias (43\%) e gêneros (76\%) monoespecíficos ocorreram em grande número.

Considerando somente o componente arbóreo, registraram-se 44 espécies (tabela 2) que corresponderam a $24 \%$ da flora total. Do conjunto de 24 famílias desse componente, 16 (67\%) apresentaram uma única espécie. Fabaceae, Myrtaceae e Vochysiaceae foram as famílias mais representativas com oito, seis e três espécies, respectivamente. Foram registradas 13 famílias $(25 \%$ do total de famílias) com ocorrência exclusiva no componente arbóreo. O componente não arbóreo (arbustos + ervas + trepadeiras + epífitas) correspondeu a $76 \%$ da flora total, abrangendo 138 espécies (tabela 2). Do 
Tabela 1. Espécies registradas na área de cerrado sensu stricto em Botucatu, SP. Hábito: arbusto (ab), árvore (av), erva (ev), epífita (ep), trepadeira (tr). Coletores: Déstro, G.F.G. \& Ishara, K.L.

Table 1. Species recorded in an area of "cerrado" sensu stricto in Botucatu, SP. Habit: shrub (ab), tree (av), herb (ev), epiphyte (ep), vine (tr). Collectors: Déstro, G.F.G. \& Ishara, K.L.

\begin{tabular}{|c|c|c|c|}
\hline Espécie & Nome popular & Hábito & № BOTU \\
\hline \multicolumn{4}{|l|}{ PTERIDÓFITAS } \\
\hline \multicolumn{4}{|l|}{ POLYPODIACEAE } \\
\hline Microgramma squamulosa (Kaulf.) de la Sota & - & ep & 24744 \\
\hline Pleopeltis angusta (Humb. \& Bonpl.) ex Willd. & polipódio-estreito & ep & 24745 \\
\hline Polypodium hirsutissimum Raddi & polipódio-hirsutíssimo & ep & 24746 \\
\hline Polypodium latipes Langsd. \& Fisch. & polipódio-de-pé-largo & ev & 24747 \\
\hline \multicolumn{4}{|l|}{ SCHYZAEACEAE } \\
\hline Anemia flexuosa (Savigny) Sw. & - & ev & 24768 \\
\hline \multicolumn{4}{|l|}{ ANGIOSPERMAS } \\
\hline \multicolumn{4}{|l|}{ ANNONACEAE } \\
\hline Duguetia furfuracea (A. St.-Hil.) Benth. \& Hook.f. & pinha-do-campo & $\mathrm{ab}$ & 24518 \\
\hline \multicolumn{4}{|l|}{ APOCYNACEAE } \\
\hline Aspidosperma tomentosum Mart. & peroba-do-campo & av & 24519 \\
\hline Blepharodon bicuspidatum E. Fourn. & - & $\operatorname{tr}$ & 24520 \\
\hline Ditassa obcordata Mart. & - & $\operatorname{tr}$ & 24521 \\
\hline Mandevilla illustris (Vell.) Woodson & jalapa & ev & 24522 \\
\hline Mandevilla velutina $\mathrm{K}$. Schum. & jalapa & ev & 24523 \\
\hline Temnadenia violacea (Vell.) Miers. & - & $\operatorname{tr}$ & 24524 \\
\hline \multicolumn{4}{|l|}{ ARALIACEAE } \\
\hline Schefflera vinosa (Cham. \& Schltdl.) Frodin \& Fiaschi & mandioqueira & av & 24525 \\
\hline \multicolumn{4}{|l|}{ ARECACEAE } \\
\hline Allagoptera campestris (Mart.) Kuntze & buri-do-campo & ev & 24528 \\
\hline \multicolumn{4}{|l|}{ ASTERACEAE } \\
\hline Acanthospermum australe (Loelf.) O. Kuntze & carrapichinho & ev & 24529 \\
\hline Achyrocline satureoides (Lam.) DC. & macela & ev & 24530 \\
\hline Baccharis dracunculifolia DC. & alecrim-do-campo & $\mathrm{ab}$ & 24532 \\
\hline Baccharis pseudotenuifolia I. L. Teodoro & alecrim-de-folha-fina & $\mathrm{ab}$ & 24531 \\
\hline Baccharis trimera DC. & carqueja-amarga & $\mathrm{ab}$ & 24533 \\
\hline Bidens gardneri Baker & picão & ev & 24535 \\
\hline Chresta sphaerocephala DC. & chapéu-de-couro & $a b$ & 24536 \\
\hline Eupatorium debeauxii B. L. Rob. & eupatório & ev & 24542 \\
\hline Eupatorium intermedium DC. & eupatório & $\mathrm{ab}$ & 24537 \\
\hline Eupatorium odoratum L. & cruzadinha & $\mathrm{ab}$ & 24538 \\
\hline Eupatorium vauthierianum DC. & eupatório & $\mathrm{ab}$ & 24543 \\
\hline Eupatorium sp.1 & - & $\mathrm{ab}$ & 24540 \\
\hline Eupatorium sp.2 & - & $\mathrm{ab}$ & 24541 \\
\hline Gochnatia pulchra (Spreng.) Cabrera & cambarazinho & $a b$ & 24544 \\
\hline Mikania strobilifera Gardner & - & $\mathrm{ab}$ & 24546 \\
\hline Piptocarpha axillaris (Less.) Baker & - & av & 24547 \\
\hline Piptocarpha macropoda (DC.) Baker & - & av & 24548 \\
\hline Piptocarpha rotundifolia (Less.) Baker & solidão & av & 24549 \\
\hline Trixis divaricata (Kunth) Spreng. & solidônia & $a b$ & 24552 \\
\hline Vernonia bardanoides Less. & - & $\mathrm{ab}$ & 24556 \\
\hline Vernonia chamissonis Less. & cambarazinho & $\mathrm{ab}$ & 24553 \\
\hline
\end{tabular}


continuação

\begin{tabular}{|c|c|c|c|}
\hline Espécie & Nome popular & Hábito & № BOTU \\
\hline Vernonia cognata Less. & assa-peixe-roxo & $a b$ & 24557 \\
\hline Vernonia elegans Gardner & - & $a b$ & 24554 \\
\hline Vernonia geminata Kunth. & - & $a b$ & 24558 \\
\hline Vernonia platensis (Spreng.) Less. & - & $a b$ & 24560 \\
\hline Vernonia polyanthes Less. & assa-peixe & $a b$ & 24561 \\
\hline Vernonia tweediana Baker & assa-peixe & $a b$ & 24563 \\
\hline \multicolumn{4}{|l|}{ BIGNONIACEAE } \\
\hline Anemopaegma glaucum Mart. ex DC. & catuaba & $a b$ & 24564 \\
\hline Arrabidaea pulchella (Cham.) Bureau & - & $\operatorname{tr}$ & 24565 \\
\hline Arrabidaea samydoides (Cham.) Sandw. & cipó-camarão & $\operatorname{tr}$ & 24566 \\
\hline Jacaranda oxyphylla Cham. & caroba-de-são-paulo & $\mathrm{ab}$ & 24567 \\
\hline Memora axillaris K. Schum. & ciganinha & $a b$ & 24568 \\
\hline Pyrostegia venusta Miers & cipó-de-são-joão & $\operatorname{tr}$ & 24571 \\
\hline Tabebuia ochracea (Cham.) Standl. & ipê-amarelo & av & 24572 \\
\hline \multicolumn{4}{|l|}{ BORAGINACEAE } \\
\hline Cordia monosperma (Jacq.) Roem. \& Schult. & moleque-duro & $a b$ & 24573 \\
\hline Tournefortia paniculata Cham. & marmelinho & $a b$ & 24574 \\
\hline \multicolumn{4}{|l|}{ BROMELIACEAE } \\
\hline Aechmea bromeliifolia (Rudge) Baker & bromélia & ep & 24575 \\
\hline \multicolumn{4}{|l|}{ CARYOCARACEAE } \\
\hline Caryocar brasiliense Cambess. & pequi-do-cerrado & $a b$ & 24576 \\
\hline \multicolumn{4}{|l|}{ CELASTRACEAE } \\
\hline Plenckia populnea Reissek & mangabeira-brava & av & 24577 \\
\hline Tontelea micrantha (Mart. ex Schult.) A. C. Sm. & bacupari-do-cerrado & $\mathrm{ab}$ & 24578 \\
\hline \multicolumn{4}{|l|}{ CHRYSOBALANACEAE } \\
\hline Couepia grandiflora (Mart. \& Zucc.) Benth. ex Hook. f. & oiti-do-sertão & av & 24580 \\
\hline \multicolumn{4}{|l|}{ CLUSIACEAE } \\
\hline Kielmeyera rubriflora Cambess. & para-tudo & av & 24581 \\
\hline Kielmeyera variabilis Mart. & malva-do-campo & $a b$ & 24584 \\
\hline \multicolumn{4}{|l|}{ COMMELINACEAE } \\
\hline Commelina erecta $\mathrm{L}$. & trapoeraba-azul & ev & 24585 \\
\hline \multicolumn{4}{|l|}{ CONVOLVULACEAE } \\
\hline Evolvulus nummularius Nutt. & - & ev & 24586 \\
\hline Ipomoea delphinioides Choisy & ipoméia & ev & 24589 \\
\hline Merremia digitata (Spreng.) Hallier f. & - & ev & 24590 \\
\hline Merremia macrocalyx (Ruiz \& Pav.) O’Donell & jitirana & $\operatorname{tr}$ & 24591 \\
\hline \multicolumn{4}{|l|}{ CUCURBITACEAE } \\
\hline Cayaponia espelina (Silva Manso) Cogn. & espelina-verdadeira & $\operatorname{tr}$ & 24593 \\
\hline Momordica charantia $\mathrm{L}$. & melão-de-são-caetano & $\operatorname{tr}$ & 24594 \\
\hline \multicolumn{4}{|l|}{ CYPERACEAE } \\
\hline Cyperus sp. & - & $\mathrm{ev}$ & 24595 \\
\hline Rhynchospora sp. & - & ev & 24597 \\
\hline \multicolumn{4}{|l|}{ DILLENIACEAE } \\
\hline Davilla elliptica St. Hil. & cipó-vermelho & $a b$ & 24598 \\
\hline \multicolumn{4}{|l|}{ EBENACEAE } \\
\hline Diospyros hispida A. DC. & caqui-do-cerrado & av & 24601 \\
\hline \multicolumn{4}{|l|}{ ERYTHROXYLACEAE } \\
\hline Erythroxylum campestre A. St.-Hil. & mercúrio-do-campo & $a b$ & 24606 \\
\hline
\end{tabular}


continuação

\begin{tabular}{|c|c|c|c|}
\hline Espécie & Nome popular & Hábito & № BOTU \\
\hline Erythroxylum cuneifolium (Mart.) O. E. Schulz. & fruta-de-pomba & $a b$ & 24608 \\
\hline Erythroxylum suberosum A. St.-Hil. & galinha-choca & $\mathrm{ab}$ & 24610 \\
\hline Erythroxylum tortuosum Mart. & galinha-choca & $a b$ & 24614 \\
\hline \multicolumn{4}{|l|}{ EUPHORBIACEAE } \\
\hline Croton glandulosus L. & canela-de-seriema & $\mathrm{ab}$ & 24615 \\
\hline Dalechampia triphylla Lam. & - & $\operatorname{tr}$ & 24616 \\
\hline Manihot caerulescens Pohl & mandioca-brava & $\mathrm{ab}$ & 24617 \\
\hline Manihot hilariana Baill. & - & ev & 24618 \\
\hline Pera glabrata (Schott) Baill. & pimenteira & av & 24619 \\
\hline Sebastiania commersoniana (Baill.) L.B. Sm. \& Dows & branquilho & av & 24622 \\
\hline Sebastiania serrulata Muell. Arg. & - & ev & 24620 \\
\hline \multicolumn{4}{|l|}{ FABACEAE - CAESALPINIOIDEAE } \\
\hline Bauhinia rufa (Bong.) Steud. & pata-de-vaca & av & 24632 \\
\hline \multicolumn{4}{|l|}{ Chamaecrista desvauxii var. brevipes (Benth.) H. S. } \\
\hline Irwin \& Barneby & sene & $\mathrm{ab}$ & 24623 \\
\hline \multicolumn{4}{|l|}{ Chamaecrista desvauxii var. langsdorffii (Kunth } \\
\hline Chamaecrista flexuosa (L.) Greene & mimosa & $\mathrm{ab}$ & 24625 \\
\hline Hymenaea stigonocarpa Mart. ex Hayne & jatobá & av & 24627 \\
\hline Senna bicapsularis (L.) Roxb. & pau-de-cachimbo & $\mathrm{ab}$ & 24628 \\
\hline Senna occidentalis (L.) Link & fedegoso & $\mathrm{ab}$ & 24629 \\
\hline Senna rugosa (G. Don.) H. S. Irwin \& Barneby & boi-gordo & $a b$ & 24630 \\
\hline \multicolumn{4}{|l|}{ FABACEAE - FABOIDEAE } \\
\hline Acosmium subelegans (Mohlenbr.) Yakovlev & amendoim-falso & av & 24634 \\
\hline Crotalaria unifoliolata Benth. & - & $\mathrm{ab}$ & 24636 \\
\hline Dalbergia miscolobium Benth. & anileira & av & 24637 \\
\hline Desmodium discolor Vogel & amores & $a b$ & 24638 \\
\hline Eriosema longifolium Benth. & - & ev & 24640 \\
\hline Glycine wightii (Grahm ex Wight \& Arn.) Verdc. & soja-perene & $\operatorname{tr}$ & 24641 \\
\hline Machaerium acutifolium Vogel & jacarandá-do-campo & av & 24642 \\
\hline Platypodium elegans Vogel & amendoim-do-campo & av & 24644 \\
\hline Rhynchosia melanocarpa J. W. Grear & olho-de-cabra & $\operatorname{tr}$ & 24645 \\
\hline Stylosanthes acuminata M. B. Ferreira \& Souza Costa & meladinho & ev & 24646 \\
\hline Zornia reticulata $\mathrm{Sm}$ & alfafa-do-campo & ev & 24647 \\
\hline \multicolumn{4}{|l|}{ FABACEAE - MIMOSOIDEAE } \\
\hline Anadenanthera falcata (Benth.) Speg. & angico-do-cerrado & av & 24648 \\
\hline Mimosa bimucronata (DC.) O. Kuntze & maricá & $\mathrm{ab}$ & 24651 \\
\hline Mimosa dolens subsp. acerba (Benth.) Barneby & juquiri & $\mathrm{ab}$ & 24652 \\
\hline Mimosa dolens subsp. rigida (Benth.) Barneby & juquiri & $\mathrm{ab}$ & 24654 \\
\hline Stryphnodendron adstringens (Mart.) Coville & barbatimão & av & 24657 \\
\hline \multicolumn{4}{|l|}{ IRIDACEAE } \\
\hline Trimezia juncifolia (Klatt.) Benth. \& Hook. f. & ruibarbo-amarelo & ev & 24658 \\
\hline \multicolumn{4}{|l|}{ LAMIACEAE } \\
\hline Aegiphila lhotszkyana Cham. & tamanqueira & $a b$ & 24660 \\
\hline Eriope crassipes Benth. & - & ev & 24664 \\
\hline Hypenia macrantha (A. St.-Hil. ex Benth.) Harley & - & ev & 24665 \\
\hline Hyptis villosa Pohl ex Benth. & hortelã-do-cerrado & $\mathrm{ab}$ & 24666 \\
\hline Peltodon tomentosus Pohl & - & ev & 24667 \\
\hline \multicolumn{4}{|l|}{ LAURACEAE } \\
\hline Ocotea corymbosa (Meisn.) Mez & canelinha & av & 24668 \\
\hline Ocotea pulchella (Nees) Mez & canelinha & av & 24670 \\
\hline
\end{tabular}


continuação

\begin{tabular}{|c|c|c|c|}
\hline Espécie & Nome popular & Hábito & № BOTU \\
\hline \multicolumn{4}{|l|}{ LYTHRACEAE } \\
\hline Lafoensia pacari A. St.-Hil. & dedaleiro & av & 24673 \\
\hline \multicolumn{4}{|l|}{ MALPIGHIACEAE } \\
\hline Banisteriopsis campestris (A. Juss.) Little & cipó-prata & $a b$ & 24677 \\
\hline Banisteriopsis oxyclada (A. Juss.) B. Gates & - & $\operatorname{tr}$ & 24678 \\
\hline Byrsonima coccolobifolia Kunth & murici-pequeno & $a b$ & 24680 \\
\hline Byrsonima intermedia A. Juss. & canjica & $a b$ & 24682 \\
\hline Byrsonima verbascifolia (L.) DC. & murici & av & 24683 \\
\hline Heteropterys umbellata A. Juss. & - & $a b$ & 24684 \\
\hline \multicolumn{4}{|l|}{ MALVACEAE } \\
\hline Eriotheca gracilipes (K. Schum.) A. Robyns & imbiriçu & av & 24685 \\
\hline Peltaea speciosa (Kunth) Standl. & - & $a b$ & 24687 \\
\hline Sida glaziovii K. Schum. & guanxuma & ev & 24688 \\
\hline Waltheria indica $\mathrm{L}$. & malva-branca & $\mathrm{ab}$ & 24689 \\
\hline \multicolumn{4}{|l|}{ MELASTOMATACEAE } \\
\hline Leandra aurea Cogn. & - & $a b$ & 24691 \\
\hline Miconia albicans (Sw.) Triana & quaresmeira-branca & $a b$ & 24692 \\
\hline Miconia langsdorffii Cogn. & jacatirão & $a b$ & 24693 \\
\hline Miconia ligustroides (DC.) Naudin & vassoura-preta & $a b$ & 24694 \\
\hline Tibouchina gracilis (Bonpl.) Cogn. & quaresmeirinha-do-brejo & $a b$ & 24698 \\
\hline Tibouchina stenocarpa (DC.) Cogn. & quaresmeira & av & 24699 \\
\hline \multicolumn{4}{|l|}{ MYRSINACEAE } \\
\hline Rapanea guianensis Aubl. & capororoca-comum & av & 24703 \\
\hline Rapanea umbellata (Mart.) Mez & capororoca-verdadeira & av & 24705 \\
\hline \multicolumn{4}{|l|}{ MYRTACEAE } \\
\hline Blepharocalyx salicifolius (Kunth) O. Berg & murta & av & 24708 \\
\hline Campomanesia pubescens (DC.) O. Berg & guabiroba & $a b$ & 24710 \\
\hline Eugenia bimarginata DC. & aperta-goela & $a b$ & 24712 \\
\hline Eugenia obversa O. Berg & fruta-de-perdiz & $\mathrm{ab}$ & 24711 \\
\hline Myrcia bella Cambess. & cambuí & av & 24713 \\
\hline Myrcia guianensis DC. & guamirim-vermelho & av & 24715 \\
\hline Myrcia lingua (O. Berg.) Mattos \& D. Legrand & brasa-viva & av & 24720 \\
\hline Myrcia multiflora (Lam.) DC. & cambuí & av & 24722 \\
\hline Psidium cinereum Mart. ex DC. & araçá & $a b$ & 24724 \\
\hline Psidium incanescens Mart. ex DC. & araçá & $\mathrm{ab}$ & 24725 \\
\hline Psidium pohlianum O. Berg & - & av & 24726 \\
\hline \multicolumn{4}{|l|}{ NYCTAGINACEAE } \\
\hline Guapira noxia (Netto) Lundell & maria-mole & av & 24728 \\
\hline Guapira opposita (Vell.) Reitz. & - & av & 24730 \\
\hline \multicolumn{4}{|l|}{ OCHNACEAE } \\
\hline Ouratea spectabilis (Mart.) Engl. & batiputá & av & 24731 \\
\hline \multicolumn{4}{|l|}{ ORCHIDACEAE } \\
\hline Epidendrum elongatum Jacq. & boca-de-dragão & ev & 24734 \\
\hline Rodriguezia decora (Lem.) Rchb. f. & - & ev & 24735 \\
\hline \multicolumn{4}{|l|}{ OROBANCHACEAE } \\
\hline Esterhazya splendida J. C. Mikan & embiri & $\mathrm{ab}$ & 24736 \\
\hline \multicolumn{4}{|l|}{ PASSIFLORACEAE } \\
\hline Passiflora alata Curtis & maracujá-açu & $\operatorname{tr}$ & 24737 \\
\hline Passiflora suberosa $\mathrm{L}$. & maracujazinho & $\operatorname{tr}$ & 24738 \\
\hline
\end{tabular}


continuação

\begin{tabular}{|c|c|c|c|}
\hline Espécie & Nome popular & Hábito & № BOTU \\
\hline \multicolumn{4}{|l|}{ POACEAE } \\
\hline Andropogon leucostachyus Kunth & capim-membeca & ev & 24739 \\
\hline Eragrostis maypurensis (Kunth) Steud. & - & ev & 24740 \\
\hline Lasiacis ligulata Hitchc. \& Chase & - & ev & 24741 \\
\hline Melinis minutiflora P. Beauv. & capim-gordura & ev & 24742 \\
\hline Tristachya leiostachya Nees & capim-flechinha & ev & 24743 \\
\hline \multicolumn{4}{|l|}{ ROSACEAE } \\
\hline Rubus brasiliensis Mart. & amora-branca & $\mathrm{ab}$ & 24748 \\
\hline \multicolumn{4}{|l|}{ RUBIACEAE } \\
\hline Alibertia concolor (Cham.) K. Schum. & marmeladinha & $a b$ & 24750 \\
\hline Borreria alata (Aubl.) DC. & erva-quente & ev & 24753 \\
\hline Coccocypselum lanceolatum (Ruiz \& Pav.) Pers. & piririca & ev & 24754 \\
\hline Declieuxia fruticosa (Willd. ex Roem. \& Schult.) Kuntze & - & ev & 24756 \\
\hline Palicourea rigida Kunth & douradinha & $a b$ & 24758 \\
\hline Psychotria sessilis (Vell.) Muell. Arg. & - & $a b$ & 24759 \\
\hline Tocoyena formosa (Cham. \& Schltdl.) K. Schum. & jenipapo-bravo & $\mathrm{ab}$ & 24760 \\
\hline \multicolumn{4}{|l|}{ SAPINDACEAE } \\
\hline Serjania erecta Radlk. & cipó-de-timbó & $\mathrm{ab}$ & 24761 \\
\hline Serjania laroutteana D. Dietr. & - & $\operatorname{tr}$ & 24764 \\
\hline Serjania sp. & & $\operatorname{tr}$ & 24763 \\
\hline \multicolumn{4}{|l|}{ SAPOTACEAE } \\
\hline Pouteria torta (Mart.) Radlk. & abiu-do-cerrado & av & 24766 \\
\hline \multicolumn{4}{|l|}{ SMILACACEAE } \\
\hline Smilax polyantha Griseb. & - & $\operatorname{tr}$ & 24769 \\
\hline \multicolumn{4}{|l|}{ SOLANACEAE } \\
\hline Solanum aculeatissimum Jacq. & joá & $a b$ & 24773 \\
\hline Solanum americanum Mill. & maria-pretinha & $a b$ & 24774 \\
\hline Solanum erianthum D. Don. & fumo-bravo & $a b$ & 24775 \\
\hline Solanum lacerdae Dusén & uvá-do-mato & $a b$ & 24779 \\
\hline Solanum lycocarpum A. St.-Hil. & lobeira & $a b$ & 24777 \\
\hline Solanum paniculatum L. & jurubeba & $a b$ & 24772 \\
\hline Solanum variabile Mart. & jurubeba-falsa & $\mathrm{ab}$ & 24778 \\
\hline \multicolumn{4}{|l|}{ STYRACACEAE } \\
\hline Styrax ferrugineus Nees \& Mart. & laranjinha-do-campo & av & 24781 \\
\hline \multicolumn{4}{|l|}{ SYMPLOCACEAE } \\
\hline Symplocos lanceolata (Mart.) A. DC. & capororoca-falsa & av & 24782 \\
\hline \multicolumn{4}{|l|}{ THYMELAEACEAE } \\
\hline Daphnopsis utilis Warm. & embira-branca & av & 24784 \\
\hline \multicolumn{4}{|l|}{ TURNERACEAE } \\
\hline Turnera hilaireana Urb. & vassourinha & ev & 24785 \\
\hline \multicolumn{4}{|l|}{ VERBENACEAE } \\
\hline Lippia lupulina Cham. & rosa-do-campo & ev & 24786 \\
\hline Lippia velutina Schauer & - & $\mathrm{ab}$ & 24787 \\
\hline \multicolumn{4}{|l|}{ VITACEAE } \\
\hline Cissus erosa Rich. & cipó-de-fogo & $\operatorname{tr}$ & 24788 \\
\hline \multicolumn{4}{|l|}{ VOCHYSIACEAE } \\
\hline Qualea grandiflora Mart. & pau-terra & av & 24790 \\
\hline Qualea multiflora Mart. & pau-terra-do-campo & av & 24791 \\
\hline Vochysia tucanorum Mart. & pau-de-tucano & av & 24793 \\
\hline
\end{tabular}


total de 38 famílias desse componente, apenas 15 (40\%) apresentaram uma única espécie. As famílias mais representativas nesse conjunto foram Asteraceae (24 espécies), Fabaceae (14) e Rubiaceae (sete). Foram registradas 27 famílias (53\% do total) com ocorrência exclusiva no componente não arbóreo.

O diagrama de perfil da vegetação estudada (figura 1) revela a ocorrência de um estrato lenhoso, constituído por árvores de pequeno a médio porte (de 3 a $7 \mathrm{~m}$ de altura), arbustos e subarbustos com caules múltiplos e o estrato herbáceo, composto por ervas com várias formas de crescimento, revestindo descontinuamente o solo sob as copas do estrato lenhoso. No trecho selecionado para a elaboração do diagrama, nota-se um dossel praticamente contínuo caracterizando um cerrado denso, com fisionomia florestal. A ocorrência do estrato herbáceo descontínuo provavelmente resulta da modificação do microclima causada pela presença deste dossel.

Tabela 2. Número de espécies, gêneros e famílias por hábito registrados na área de cerrado sensu stricto em Botucatu, SP.

Table 2. Number of species, genus and family per habit recorded in a area of "cerrado" sensu stricto in Botucatu, SP.

\begin{tabular}{lccccc}
\hline & Árvores & Arbustos & Ervas & Trepadeiras & Epífitas \\
\hline Espécies & 44 & 78 & 37 & 19 & 4 \\
Gêneros & 35 & 47 & 36 & 16 & 4 \\
Famílias & 24 & 22 & 18 & 11 & 2 \\
Espécies invasoras & 0 & 15 & 7 & 4 & 0 \\
\hline
\end{tabular}
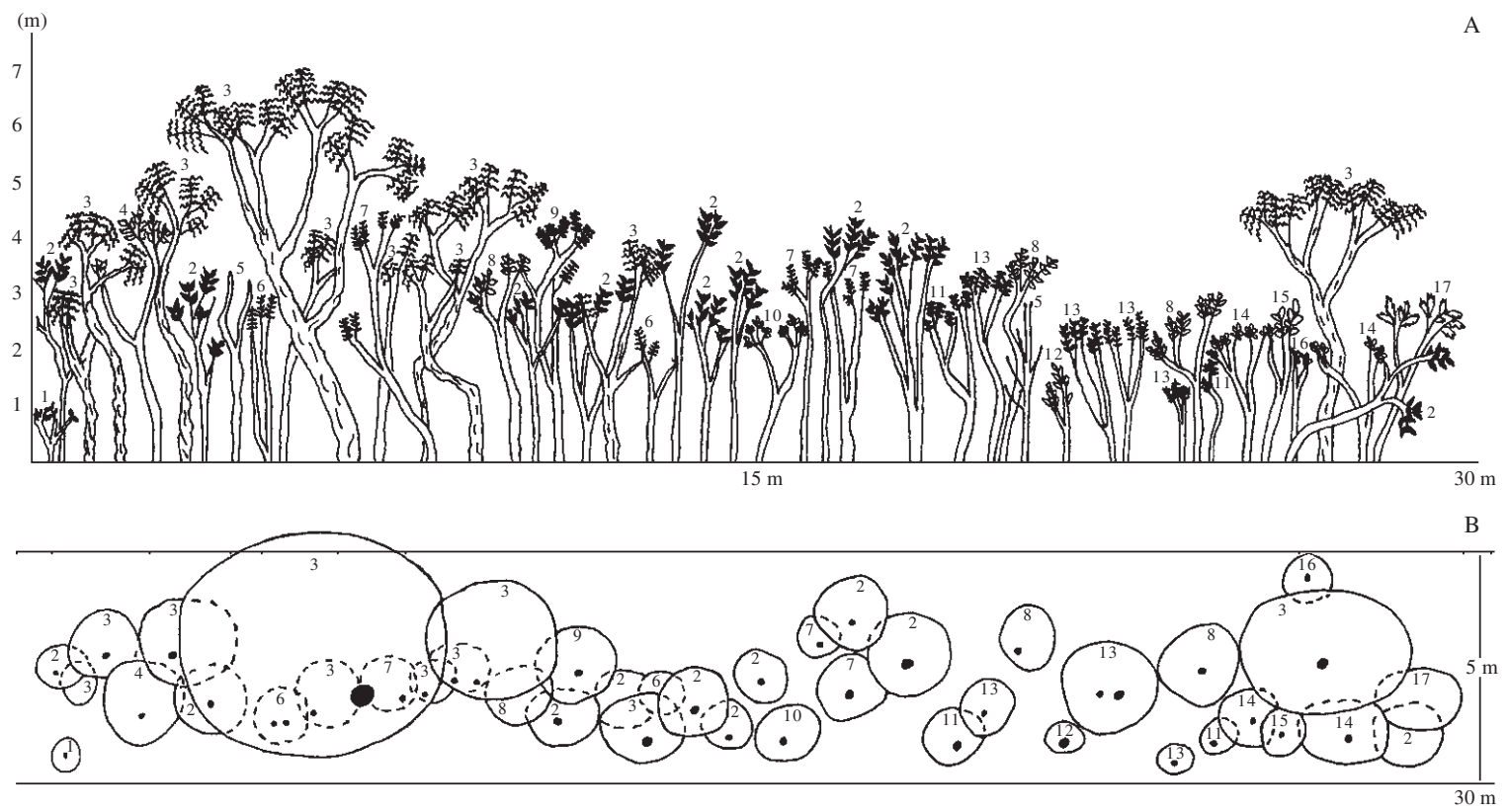

Figura 1. Diagrama de perfil (A) e projeções das copas (B) representando o componente arbustivo-arbóreo em área de cerrado sensu stricto no município de Botucatu, SP. 1. Erythroxylum suberosum. 2. Tibouchina stenocarpa. 3. Anadenanthera falcata. 4. Rapanea umbellata. 5. Morta. 6. Campomanesia pubescens. 7. Myrcia lingua. 8. Ouratea spectabilis. 9. Dalbergia miscolobium. 10. Tabebuia ochracea. 11. Acosmium subelegans. 12. Eupatorium vauthierianum. 13. Myrcia guianensis. 14. Schefflera vinosa. 15. Guapira noxia. 16. Rapanea guianensis. 17. Piptocarpha axillaris.

Figure 1. Profile diagram (A) and canopy projections (B) representing the woody component in a "cerrado" sensu stricto area in Botucatu, SP. 1. Erythroxylum suberosum. 2. Tibouchina stenocarpa. 3. Anadenanthera falcata. 4. Rapanea umbellata. 5. Dead tree. 6. Campomanesia pubescens. 7. Myrcia lingua. 8. Ouratea spectabilis. 9. Dalbergia miscolobium. 10. Tabebuia ochracea. 11. Acosmium subelegans. 12. Eupatorium vauthierianum. 13. Myrcia guianensis. 14. Schefflera vinosa. 15. Guapira noxia. 16. Rapanea guianensis. 17. Piptocarpha axillaris. 
A matriz para a análise de similaridade florística englobou 640 espécies registradas nas seis localidades comparadas (tabela 3), das quais $388(61 \%)$, por ocorrerem em apenas um local, foram desconsideradas. Somente 138 espécies (22\%) ocorreram em mais de 50\% dos locais e apenas 13 espécies (2\%) foram registradas em todas as localidades comparadas, quais sejam: Anadenanthera falcata, Machaerium acutifolium (Fabacaeae), Byrsonima coccolobifolia, B. verbascifolia (Malpighiaceae), Caryocar brasiliense (Caryocaraceae), Schefflera vinosa (Araliaceae), Miconia albicans (Melastomataceae), Myrcia bella (Myrtaceae), Ouratea spectabilis (Ochnaceae), Qualea grandiflora, $Q$. multiflora, Vochysia tucanorum (Vochysiaceae) e Styrax ferrugineus (Styracaceae).

Os índices de similaridade calculados produziram valores baixos, não superiores a 0,48 para o Índice de Jaccard (tabela 4) e 0,65 para o Índice de Sørensen (tabela 5). Com estes dois índices, a área de estudo apresentou-se mais similar aos cerrados de Agudos e Botucatu. A análise de agrupamento produziu resultados semelhantes (figura 2), formando-se dois subgrupos, um dos quais incluiu apenas as áreas de Assis, e outro contendo as demais localidades; neste último, houve maior similaridade entre as áreas de Agudos e Botucatu, sendo a área inventariada no presente estudo a menos similar do grupo.

Tabela 3. Áreas de cerrado no Estado de São Paulo comparadas com o presente estudo.

Table 3. Areas of "cerrado" in São Paulo State compared with this study.

\begin{tabular}{|c|c|c|c|c|c|c|}
\hline Sigla & Município & Fisionomia & Coordenadas geográficas & Clima & Altitude $(\mathrm{m})$ & Referência \\
\hline BOTU & Botucatu & $\begin{array}{l}\text { cerrado } \\
\text { sensu stricto }\end{array}$ & $22^{\circ} 57^{\prime} 34^{\prime \prime} \mathrm{S} 48^{\circ} 31^{\prime} 20^{\prime \prime} \mathrm{W}$ & $\mathrm{Cfa}$ & 830 & Presente estudo \\
\hline ASSc & Assis & $\begin{array}{l}\text { cerrado } \\
\text { sensu stricto }\end{array}$ & $\begin{array}{l}22^{\circ} 33^{\prime} 65^{\prime \prime} \text { a } 22^{\circ} 36^{\prime} 68^{\prime \prime} \mathrm{S} \\
50^{\circ} 23^{\prime} 00^{\prime \prime} \text { a } 50^{\circ} 22^{\prime} 29^{\prime \prime} \mathrm{W}\end{array}$ & Cwa & 520 a 590 & Durigan et al. (1999) \\
\hline ASSC & Assis & cerradão & $\begin{array}{l}22^{\circ} 33^{\prime} 65^{\prime \prime} \text { a } 22^{\circ} 36^{\prime} 68^{\prime \prime} \mathrm{S} \\
50^{\circ} 23^{\prime} 00^{\prime \prime} \text { a } 50^{\circ} 22^{\prime} 29^{\prime \prime} \mathrm{W}\end{array}$ & Cwa & 520 a 590 & Durigan et al. (1999) \\
\hline AGUD & Agudos & $\begin{array}{l}\text { cerrado } \\
\text { sensu stricto }\end{array}$ & $\begin{array}{l}22^{\circ} \text { a } 23^{\circ} \mathrm{S} 49^{\circ} 30^{\prime} \text { a } \\
48^{\circ} 50^{\prime} \mathrm{W}\end{array}$ & Cwa & 550 & Bertoncini (1996) \\
\hline SRPQ & $\begin{array}{l}\text { Santa Rita do } \\
\text { Passa Quatro }\end{array}$ & $\begin{array}{l}\text { cerrado } \\
\text { sensu stricto }\end{array}$ & $21^{\circ} 43^{\prime} \mathrm{S} 47^{\circ} 35^{\prime} \mathrm{W}$ & Cwa & 600 & Weiser \& Godoy (2001) \\
\hline BOT & Botucatu & cerradão & $22^{\circ} 48^{\prime} \mathrm{S} 48^{\circ} 17^{\prime} \mathrm{W}$ & Cfa & 500 & Bicudo (1987) \\
\hline
\end{tabular}

Tabela 4. Índice de Jaccard entre seis áreas de cerrado sensu lato no Estado de São Paulo. As áreas encontram-se na tabela 3 .

Table 4. Jaccard Index among six areas of "cerrado" sensu lato in the São Paulo State. Areas are given in table 3.

\begin{tabular}{lcccccc}
\hline & BOTU & ASSc & ASSC & AGUD & SRPQ & BOT \\
\hline BOTU & $*$ & 0,3190 & 0,2460 & 0,3920 & 0,3226 & 0,3529 \\
ASSc & & $*$ & 0,4673 & 0,3756 & 0,3333 & 0,4247 \\
ASSC & & & $*$ & 0,2621 & 0,2291 & 0,2350 \\
AGUD & & & & $*$ & 0,4061 & 0,4815 \\
SRPQ & & & & & $*$ & 0,3483 \\
BOT & & & & & & $*$ \\
\hline
\end{tabular}

Tabela 5. Índice de Sørensen entre seis áreas de cerrado sensu lato no Estado de São Paulo. As áreas encontram-se na tabela 3 .

Table 5. Sørensen Index among six areas of "cerrado" sensu lato in the São Paulo State. Areas are given in table 3.

\begin{tabular}{lcccccc}
\hline & BOTU & ASSc & ASSC & AGUD & SRPQ & BOT \\
\hline BOTU & $*$ & 0,4838 & 0,3948 & 0,5633 & 0,4878 & 0,5217 \\
ASSc & & $*$ & 0,6370 & 0,5461 & 0,5000 & 0,5962 \\
ASSC & & & $*$ & 0,4154 & 0,3727 & 0,3806 \\
AGUD & & & & $*$ & 0,5776 & 0,6500 \\
SRPQ & & & & & $*$ & 0,5167 \\
BOTU & & & & & & $*$ \\
\end{tabular}




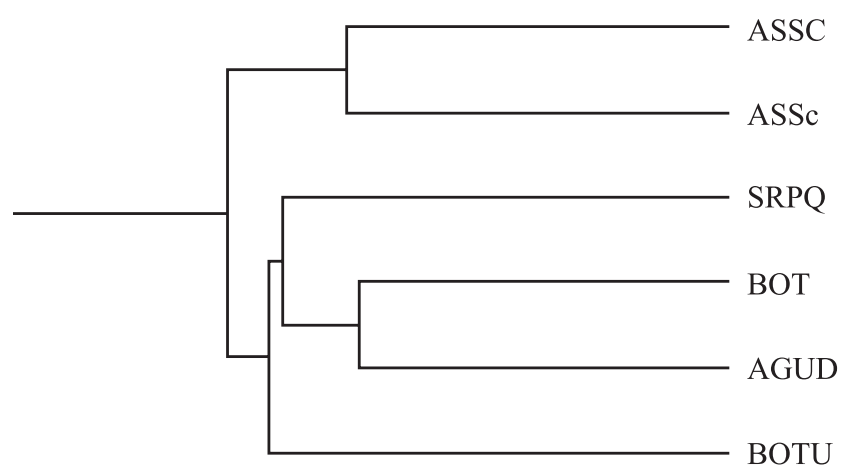

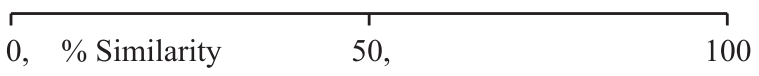

Figura 2. Análise de agrupamento (UPGMA), utilizando o índice de similaridade de Jaccard, para seis áreas de cerrado sensu lato no estado de São Paulo. As áreas encontram-se na tabela 3 .

Figure 2. Cluster analysis (UPGMA) considering Jaccard's Index among six "cerrado" sensu lato areas in São Paulo State. Areas are given in table 3.

\section{Discussão}

Analisando a flora como um todo, as famílias com maior riqueza de espécies encontradas neste levantamento também são as mais ricas, tanto no bioma Cerrado (Mendonça et al. 1998) quanto em levantamentos florísticos realizados em outros fragmentos de cerrado no Estado de São Paulo (Cavassan 2002).

As espécies encontradas correspondem a $2,4 \%$ do total daquelas compiladas para a flora vascular do bioma Cerrado (Mendonça et al. 1998) e a 5\% das espécies relatadas por Castro et al. (1999) para a flora lenhosa dos cerrados do país. Das 100 espécies lenhosas mais freqüentes nos cerrados da província florística do Sul do país, ou seja, cerrados de São Paulo, Paraná e sul de Minas Gerais (Bridgewater et al. 2004), 48 espécies (27\% do total) foram registradas no presente levantamento. Esses dados demonstram a importância do fragmento estudado, em termos de contribuição com espécies pouco frequientes, em relação aos cerrados da província sul do Brasil.

Dentre as espécies de angiospermas identificadas, $18(10 \%)$ não foram encontradas nas principais listagens para a flora do cerrado (Mendonça et al. 1998, Castro et al. 1999, Ratter et al. 2003, Durigan et al. 2004b). Algumas dessas espécies, por exemplo, Eupatorium intermedium, E. odoratum, Trixis divaricata, Dalechampia triphylla, Sebastiania commersoniana, Solanum lacerdae e Daphnopsis utilis ocorrem em áreas de vegetação litorânea ou fragmentos florestais. Segundo Durigan et al. (2003a), no Estado de São Paulo, há um gradiente florístico de cerrado para floresta estacional semidecidual, com áreas de ecótono contendo proporções variáveis de espécies de cerrado e de floresta. Considerando a proximidade da área estudada com um fragmento de floresta estacional semidecidual e a presença de espécies incomuns à vegetação de cerrado, a área de estudo poderia representar uma área de ecótono.

Vernonia e Eupatorium foram os gêneros mais ricos do componente não arbóreo, sendo que Vernonia é um dos gêneros com maior riqueza entre as Asteraceae no bioma Cerrado (Mendonça et al. 1998). Apesar da riqueza considerável registrada para o gênero Solanum, cinco espécies são reputadas invasoras: S. aculeatissimum, $S$. americanum, $S$. erianthum, $S$. lycocarpum e $S$. paniculatum (Lorenzi 1982). A presença destas espécies foi anteriormente assinalada para o bioma Cerrado (Mendonça et al. 1998). Além disso, foram encontradas outras 21 espécies invasoras distribuídas nas famílias Asteraceae, Bignoniaceae, Boraginaceae, Convolvulaceae, Euphorbiaceae, Fabaceae, Malpighiaceae, Malvaceae, Poaceae e Rubiaceae (Lorenzi 1982), correspondendo este grupo a cerca de $14 \%$ do total de espécies. Gêneros introduzidos no país (Souza \& Lorenzi 2005) também foram encontrados no local, tais como Momordica, Glycine e Melinis. Esse resultado indica que a área encontra-se alterada, tendo-se informação sobre a redução de seu tamanho e considerável interferência antrópica em épocas passadas. Mesmo sendo uma área conservada desde 2001, quando se instalou no local a empresa atualmente responsável, parte dos fragmentos de cerrado encontra-se ao redor de hortas mantidas pela empresa, o que pode ocasionar a invasão de espécies exóticas na área de vegetação nativa.

Apesar do grande número de espécies invasoras, registrou-se a presença de Turnera hilaireana, considerada uma espécie vulnerável no Estado de São Paulo (Resolução SMA - 48, 21/9/2004), indicando que áreas de cerrado de pequena extensão também devem ser integradas a programas de preservação. Somado a isso, Mandevilla illustris e Kielmeyera rubriflora, espécies de distribuição geográfica restrita no Estado paulista (Durigan et al. 2003a) também foram encontradas no local, demonstrando a importância desses pequenos fragmentos.

A representatividade das famílias com maior riqueza, tanto do componente arbóreo quanto do não arbóreo, foi coincidente com os resultados obtidos para o cerrado de Santa Rita do Passa Quatro (Weiser \& 
Godoy 2001). Apenas 11 famílias apresentaram espécies nos componentes arbóreo e não-arbóreo, evidenciando a importância da amostragem de plantas de todos os hábitos. A proporção de espécies do componente arbóreo para o não arbóreo foi de $1: 3$, relação semelhante à encontrada na literatura para outras áreas de cerrado (Oliveira Filho \& Ratter 2002).

A comparação com a flora de áreas de cerrado no Estado de São Paulo, efetuada para estimar níveis de similaridade, evidenciou grande heterogeneidade florística, com muitas espécies ocorrendo em apenas um local, fato este também observado por Ratter et al. (2003) na comparação de diversas áreas de cerrado no país. Além disso, o total de espécies utilizadas para a comparação em cada área foi muito variável, abrangendo de 96 a 168 espécies, após a exclusão daquelas com identificação incompleta ou duvidosa e das que ocorreram em um único local.

Os níveis de similaridade florística foram baixos, mesmo entre áreas próximas. Os maiores valores obtidos para os dois índices de similaridade calculados foram muito baixos, atingindo níveis obtidos somente quando se comparam áreas muito distantes ou muito diversas, como se pode verificar em Bridgewater et al. (2004), Balduino et al. (2005) e Batalha \& Martins (2007). Há, portanto, variação significativa na composição florística das áreas comparadas, evidenciando a ocorrência de padrões florísticos regionais, a despeito do fato de todas pertencerem à mesma província de cerrado (Bridgewater et al. 2004). Resultados semelhantes foram anteriormente apontados para áreas de cerrado geograficamente próximas (Felfili \& Silva Júnior 1993, Bridgewater et al. 2004), caracterizando a grande heterogeneidade florística dessa formação (Ratter \& Dargie 1992). Segundo Durigan et al. (2003b) as relações de similaridade florística podem ser estabelecidas em função do clima, da fertilidade dos solos ou da bacia hidrográfica à qual determinada área pertence. Neste contexto, um caráter comum entre as áreas mais similares ao presente estudo refere-se ao tipo de solo, classificado como Latossolo Vermelho-Amarelo ou Latossolo Vermelho-Escuro, textura média. A formação de dois subgrupos observada na análise de agrupamento (figura 2) revela maior similaridade entre áreas geograficamente próximas, independentemente da fisionomia. Assim, por exemplo, houve maior similaridade entre o cerradão e o cerrado sensu stricto do Município de Assis, que constituíram um subgrupo bastante distinto, enquanto o segundo subgrupo constituiu-se de áreas de cerrado sensu stricto e cerradão, sendo a maioria delas geograficamente próximas. O baixo nível de similaridade entre o presente levantamento e os demais elementos de seu subgrupo pode ser atribuído à diferença de altitude associada às variações nos tipos climáticos entre as áreas comparadas (figura 2, tabela 3). Estes fatos ganham relevância quando se considera que oito espécies arbustivas (Eupatorium intermedium, E. odoratum, Mikania strobilifera, Trixis divaricata, Vernonia geminata, V. tweediana, Solanum lacerdae, S. variabile), duas arbóreas (Sebastiania commersoniana, Daphnopsis utilis), duas herbáceas (Eupatorium debeauxii, Borreria alata) e duas trepadeiras (Arrabidaea samydoides, Dalechampia triphylla), todas não invasoras, encontradas no presente levantamento, não constam nas principais listas de espécies do cerrado (Mendonça et al. 1998, Castro et al. 1999, Ratter et al. 2003, Durigan et al. 2004b). Desta forma, os resultados obtidos no presente estudo indicam a necessidade de proteger até mesmo pequenos fragmentos de vegetação nativa, para abranger de modo significativo a variabilidade florística da região.

Agradecimentos - Ao Grupo Centroflora pela permissão ao desenvolvimento do estudo nas dependências da empresa e apoio ao projeto. À Capes pela bolsa concedida à primeira autora. Aos professores Jorge Y. Tamashiro e João Semir, ambos do Departamento de Botânica do Instituto de Biologia, Unicamp, pela identificação de algumas plantas, assim como aos estudantes de pós-graduação pela identificação de determinadas famílias (A.P. SantosGonçalves: Poaceae; K.F. Rodrigues: Melastomataceae; J. Aranha: Symplocaceae; R.R. Silva: Fabaceae). À Dra. Ingrid Koch pela identificação das espécies de Apocynaceae e ao Clemente Campos, técnico do Departamento de Botânica, IBB, Unesp, pela ajuda na etapa preliminar de identificação.

\section{Referências bibliográficas}

BALDUINO, A.P.C., SOUZA, A.L., MEIRA NETO, J.A.A., SILVA, A.F. \& SILVA JÚNIOR, M. C. 2005. Fitossociologia e análise comparativa da composição florística do cerrado da flora de Paraopeba-MG. Revista Árvore 29:25-34.

BATALHA, M.A. \& MARTINS, F.R. 2007. The vascular flora of the cerrado in Emas National Park (Central Brazil): a savanna flora summarized. Brazilian Archives of Biology and Techology 50:269-277.

BERTONCINI, A.P. 1996. Composição florística e estrutura fitossociológica de uma área de cerrado no município de Agudos-SP. Dissertação de mestrado, Universidade Estadual Paulista, Botucatu.

BICUDO, L.R.H. 1987. Mapeamento dos cerrados (sensu lato) do Município de Botucatu/SP. Florística de duas áreas. Dissertação de mestrado, Universidade Estadual Paulista, Botucatu. 
BICUDO, L.R.H. 1995. Florística, fitossociologia e ciclagem de nutrientes em um cerrado no Município de BotucatuSP. Tese de doutorado, Universidade Estadual Paulista, Rio Claro.

BICUDO, L.R.H., CÉSAR, O. \& MONTEIRO, R. 1996. A comparative floristic analysis of a cerrado area in Botucatu, State of São Paulo (Brazil). Arquivos de Biologia e Tecnologia 39:685-691.

BRIDGEWATER, S., RATTER, J.A. \& RIBEIRO, J.F. 2004. Biogeographic patterns, $\beta$-diversity and dominance in the cerrado biome of Brazil. Biodiversity and Conservation 13:2295-2318.

CAMPOS, S., SILVA, M., PIROLI, E.L., CARDOSO, L.G. \& BARROS, Z.X. 2004. Evolução do uso da terra entre 1996 e 1999 no município de Botucatu-SP. Engenharia Agrícola 24:211-218.

CASTRO, A.A.J.F., MARTINS, F.R., TAMASHIRO, J.Y. \& SHEPHERD, G.J. 1999. How rich is the flora of Brazilian cerrados? Annals of the Missouri Botanical Garden 86:192-226.

CAVASSAN, O. 2002. O cerrado do Estado de São Paulo. In Eugen Warming e o cerrado brasileiro: um século depois (A.L. Klein, org.). Editora Unesp, São Paulo, p.93-106.

DURIGAN, G. 2003. Métodos para análise de vegetação arbórea. In Métodos de estudos em biologia da conservação e manejo da vida silvestre. (L. Cullen Jr., R. Rudran \& C. Valladares-Padua, org.). Editora da UFPR, Curitiba, p.455-479.

DURIGAN, G., BACIC, M.C., FRANCO, G.A.D.C. \& SIQUEIRA, M.F. 1999. Inventário florístico do cerrado na Estação Ecológica de Assis, SP. Hoehnea 26: 149-172.

DURIGAN, G., SIQUEIRA, M.F., FRANCO, G.A.D.C., BRIDGEWATER, S. \& RATTER, J.A. 2003a. The vegetation of priority areas for cerrado conservation in São Paulo State, Brazil. Edinburgh Journal of Botany 60:217-241.

DURIGAN, G., RATTER, J.A., BRIDGEWATER, S., SIQUEIRA, M.F. \& FRANCO, G.A.D.C. 2003b. Padrões fitogeográficos do cerrado paulista sob uma nova perspectiva regional. Hoehnea 30:39-51.

DURIGAN, G., FRANCO, G.A.D.C. \& SIQUEIRA, M.F. 2004a. A vegetação dos remanescentes de cerrado no Estado de São Paulo. In Viabilidade de conservação dos remanescentes de cerrado no Estado de São Paulo (M.D. Bitencourt \& R.R. Mendonça, org.). Annablume, Fapesp, São Paulo, p.29-56.

DURIGAN, G., BAITELLO, J.B., FRANCO, G.A.D.C. \& SIQUEIRA, M.F. 2004b. Plantas do cerrado paulista: imagens de uma paisagem ameaçada. Páginas e Letras Editora e Gráfica, São Paulo.

EMBRAPA. 1999. Sistema brasileiro de classificação de solos. Empresa Brasileira de Pesquisa Agropecuária, Centro Nacional de Pesquisa de Solos, Rio de Janeiro.
FELFILI, J.M. \& SILVA JÚNIOR, M.C. 1993. A comparative study of cerrado (sensu stricto) vegetation in Central Brazil. Journal of Tropical Ecology 9:277-289.

JORGE, L.A.B. \& MOREIRA, M.P. 2000. Padrões da fragmentação do habitat na Cuesta de Botucatu (SP). Ciência Florestal 10:141-157.

LEITÃO FILHO, H.F. 1992. A flora arbórea dos cerrados do Estado de São Paulo. Hoehnea 19:151-163.

LORENZI, H. 1982. Plantas daninhas do Brasil: terrestres, aquáticas, parasitas, tóxicas e medicinais. Edição do autor, Nova Odessa.

MENDONÇA, R.C., FELFILI, J.M., WALTER, B.M.T., SILVA JUNIOR, M.C., REZENDE, A.V., FILGUEIRAS, T.S. \& NOGUEIRA, P.E. 1998. Flora vascular do cerrado. In Cerrado: ambiente e flora (S.M. Sano \& S.P. Almeida, eds.). Embrapa, Planaltina, p.289-556.

MUELLER-DOMBOIS, D. \& ELLENBERG, H. 1974. Aims and methods of vegetation ecology. John Wiley \& Sons, New York.

OLIVEIRA-FILHO, A.T. \& RATTER, J.A. 2002. Vegetation physiognomies and woody flora of the Cerrado Biome. In The Cerrados of Brazil: ecology and natural history of a Neotropical Savanna (P.S. Oliveira \& R.J. Marquis, eds.). Columbia University Press, New York, p.91-120.

RESOLUÇÃO SMA - 48, de 21/9/2004. Diário Oficial, vol.114, n.179, São Paulo.

RATTER, J.A. \& DARGIE, T.C.D. 1992. An analysis of the floristic composition of 26 cerrado areas in Brazil. Edinburgh Journal of Botany 49:235-250.

RATTER, J.A., BRIDGEWATER, S. \& RIBEIRO, J.F. 2003. Analysis of the floristic composition of the Brazilian cerrado vegetation III: comparison of the woody vegetation of 376 areas. Edinburgh Journal of Botany 60:57-109.

RIBEIRO, J.F. \& WALTER, B.M.T. 1998. Fitofisionomias do bioma cerrado. In Cerrado: ambiente e flora (S.M. Sano \& S.P. Almeida, eds.). Embrapa, Brasília, p.89-166.

SILBERBAUER-GOTTSBERGER, I. \& EITEN, G. 1987. A hectare of cerrado, I. General aspects of the trees and thick-stemmed shrubs. Phyton 27:55-91.

SNEATH, P.H. \& SOKAL, R.R. 1973. Numerical taxonomy. W.H. Freeman \& Co., San Francisco.

SOUZA, V.C. \& LORENZI, H. 2005. Botânica sistemática: guia ilustrado para identificação das famílias de Angiospermas da flora brasileira, baseado em APG II. Instituto Plantarum, Nova Odessa.

SPIRANDELLI-CRUZ, E.F. 2004. Anfíbios anuros de remanescentes de mata atlântica na região de Botucatu. In Flora e fauna: um dossiê ambiental. (W. Uieda \& L.M. Paleari, org.). UNESP, São Paulo. p.91-98.

TRYON, R.M. \& TRYON, A.F. 1982. Ferns and allied plants. Springer-Verlag, New York.

WEISER, V.L. \& GODOY, S.A.P. 2001. Florística de um hectare de cerrado stricto sensu na ARIE - cerrado Péde-Gigante, Santa Rita do Passa Quatro, SP. Acta Botanica Brasilica 15:201-212. 